\title{
A Satisfaction Evaluation Model of the Rail Feeder Modes Based on SEM
}

\author{
Zhou Yun-yun ${ }^{1}$, Yu Lu-man ${ }^{1}$, Teng Jing ${ }^{2,}$ * \\ ${ }^{1}$ Institute of Rail Transit, Tongji University, Shanghai, China \\ ${ }^{2}$ Key Laboratory of Road and Traffic Engineering of the Ministry of Education, Tongji University, Shanghai, China
}

\section{Email address:}

1452142@tongji.edu.cn (Zhou Yun-yun),2014lumanyu@tongji.edu.cn (Yu Lu-man), tengjing@tongji.edu.cn (Teng Jing)

${ }^{*}$ Corresponding author

\section{To cite this article:}

Zhou Yun-yun, Yu Lu-man, Teng Jing. A Satisfaction Evaluation Model of the Rail Feeder Modes Based on SEM. Science Discovery. Vol. 6, No. 5, 2018, pp. 320-326. doi: 10.11648/j.sd.20180605.12

Received: July 28, 2018; Accepted: August 18, 2018; Published: September 18, 2018

\begin{abstract}
As the city entering the era of public transport mainly with the rail transit, the good feeder system and transfer system have become the most prominent problems in urban rail transit. High synergy between urban rail transit and transfer facilities is an inevitable requirement to optimize traffic, but there are few studies on urban rail transfer and the research content is only based on data statistics without considering the mutual influence of various factors. For more accurate evaluation of the relevant service level, this paper combines customer satisfaction and SERVQUAL theory with the structural equation, uses SmartPLS software to explore the evaluation model of urban rail transfer, and then in this paper we investigate the citizens in Shanghai to calculate the satisfaction index of bus, taxi, bicycle and pedestrian as empirical analysis. This model can analyze the relationship between different variables, gain the key factors influencing the satisfaction index, and point out the problems in the connection service, so as to provide decisions supporting the optimization of urban rail transfer. The results of the model can be used in various connection modes and can solve the remaining "last kilometer" problem of urban rail transit, which is of great significance to improve the service level and operation efficiency of rail transit.
\end{abstract}

Keywords: Integrated Transportation, Satisfaction Evaluation Model, Structural Equation Model, Rail Feeder Modes, Transportation in Shanghai

\section{基于结构方程的城轨接驳换乘满意度模型}

\author{
周芸芸 ${ }^{1}$, 俞路漫 ${ }^{2}$, 滕靖 ${ }^{*}$ \\ 1 铁道与城市轨道交通研究院, 同济大学, 上海, 中国 \\ 2 道路与交通工程教育部重点实验室, 同济大学, 上海, 中国

\section{邮箱} \\ 1452142@tongji.edu.cn (周芸芸), 2014lumanyu@tongji.edu.cn（俞路漫）, tengjing@tongji.edu.cn(滕靖)
}

摘要: 随着城市进入以轨道交通为主体的公共运输时代, 良好的馈送系统和换乘接驳系统成为目前城轨交通中最突出 的问题。城市轨道交通与换乘接驳设施的高度协同是优化交通出行的必然要求, 但城轨接驳换乘的相关研究还非常少, 研究内容也仅仅基于数据统计, 未考虑各因素的相互影响。为更准确评价轨道交通站点接驳换乘的相关服务水平, 本 文结合顾客满意度、SERVQUAL理论等, 运用SmartPLS软件探索评价城轨接驳换乘满意度的结构方程模型, 并就上海 市郊区市民出行的调查数据对公交、打车、共享单车和步行四种接驳方式进行实证分析。该模型能分析各变量的相互 关系、获得影响满意度评价的关键因素、指出接驳服务中存在的问题等，为城轨接驳优化提供决策支持。模型成果被 
能够应用于多种接驳方式, 能够解决城轨交通的遗留“最后一公里”问题, 对提轨道交通服务水平及运行效率具有重要 意义。

关键词: 综合交通运输, 满意度评价模型, 结构方程模型, 城轨接驳, 上海交通出行

\section{1. 引言}

近年来，上海城市规模不断扩大，城市轨道交通进入 了市域化、网络化运营的新时期。但是由于轨道交通在客 运服务中可达性较低, 仍然十分需要地面公交的接驳喂送。 城轨接驳意为从轨道交通站点到目的地的短距离出行, 其 效率直接影响公众出行效率和社会整体效率。顾客满意 (Customer Satisfaction, CS)[1]是指顾客对其要求已被满足 程度的感受, Richard N Cardozo[2]最早将顾客满意度引入 营销科学领域。为评价公共交通的服务水平，方蕾等 [3] 从地铁乘客满意度视角出发, 建立了地铁服务质量评价指 标体系。张双 [4]、吴绍玉 [5]等建立了地铁乘客满意度指 数模型和地铁乘客满意度评价指标体系, 确定了各级评价 指标计算方法及评价标准并且编制了地铁乘客满意度的 调查方案。金宁[6]、顾品[7]等运用顾客满意度指数模型 的研究成果, 构建了公共交通乘客满意度概念模型, 并采 用基于偏最小二乘法的结构方程模型（Partial Least Square-Structural Equation Model, PLS-SEM）量化并解释 了乘客满意度。郭陆灯等[8]用结构方程模型的方法对长春 轻轨公司提供的各项服务进行了满意度的评价。

对于城轨接驳来说, Smith R G W, Mulamoottil G[9] 统计了地铁乘客常用的接驳方式, 分析了影响乘客选择出 行方式的因素。但城轨交通接驳的形式多样复杂, 目前的 研究多只涉及单一的接驳方式, 如乔婧等[10]分析了选用 公交接驳的影响因素、周强[11]论述了公共自行车接驳的 使用特征与幸福感, 缺乏对公交、打车、共享单车、步行 等通用的满意度评价模型。本文将最小二乘法-结构方程 模型 (PLS-SEM) 引入到城轨接驳换乘系统的评价中, 结 合乘客满意度领域已有成果 [12], 根据SERVQUAL理论 [13]建立评价指标, 探索一个灵活多变、简单通用的满意 度评价模型, 可以根据需要应用于多个交通领域, 为城市 交通系统的满意度评价问题提供新的解决思路。

\section{2. 问题描述与建模}

城轨换乘满意度评价模型的主体是地铁乘客, 评价对 象是城轨换乘系统, 通常包括公交、打车、共享单车、步 行四种出行方式[14], 评价目的是了解公众的要求与意见, 优化资源配置, 使交通出行系统的改善和优化能够反映公 众的需求和利益。

\section{1. 乘客满意度指数模型}

乘客满意度是顾客满意度在交通领域的延伸与应用。 美国顾客满意度指数模型（ACSI）[5]的维度包括顾客期 望、感知质量、感知价值、顾客满意度、顾客抱怨、顾客 忠诚六个结构变量。根据预调查收集的数据, 发现乘客在
接驳换乘的短距离出行中感知价值对乘客满意度的路径 系数约为 0 , 说明感知价值对乘客满意度几乎无影响。并 且考虑到接驳换乘过程中步行等方式没有感知价值属性, 本文满意度指数模型去掉感知价值结构变量。参考莫一魁 ${ }^{[8]}$ 等的研究成果, 本文将感知质量细分为三个部分, 由此 共确定七个潜变量: 乘客期望（PE）、乘客满意（PS）、 乘客抱怨 (PC) 、乘客忠诚 (PL) 以及由感知质量细分 出设施水平 $(\mathrm{FL})$ 、运营组织 $(\mathrm{OO})$ 和服务质量 $(\mathrm{SQ})$ 。

\section{2. 基于SEM的满意度模型}

乘客期望、感知质量等结构变量并不能直接测量, 需 要可观观察的变量作为这些潜在变量的观测指标。结构方 程模型能够分析多个潜变量之间的结构关系, 又能够在分 析中处理测量误差。偏最小二乘法-结构方程模型在求解 过程中允许测量变量、潜变量为非正态分布, 而通过潜变 量与其自身测量变量以及潜变量与其他相关变量的测量 变量之间的一系列迭代进行求解 [15]。评价指标应符合公 众认知水平、方便问卷设计和制定优化方案

本文构建的结构方程模型如下, 外生潜变量乘客期望 $(\mathrm{PE}) 、 内$ 生潜变量乘客满意 $(\mathrm{PS}) 、$ 乘客抱怨 $(\mathrm{PC})$ 、 乘客忠诚 $(\mathrm{PL})$ 、设施水平 $(\mathrm{FL})$ 、运营组织 $(\mathrm{OO})$ 和 服务质量 (SQ)。本文乘客期望（PE）对应的指标是总 体期望 (x1) 和对可靠程度的期望 (x2)、乘客满意 (PS) 对应的指标是总体满意度 $(\mathrm{y} 1)$ 和与理想服务水平的差距 (y2)、乘客抱怨对应的指标包括是否抱怨 (c1) 和是否 投诉（c2）、乘客忠诚对应是否推荐他人使用（11）和再 次使用 (12)。感知质量的三部分比较灵活, 设施水平 (FL) 包含站点可达性 (F1)、换乘便捷性 (F2), 运营组织 (OO) 对应等候时间 $(\mathrm{O} 1)$ 、动态信息 $(\mathrm{O} 2)$ 和服务范围 $(\mathrm{O} 3)$ 、 服务质量 $(\mathrm{SQ})$ 对应指标有安全性 $(\mathrm{S} 1)$ 、舒适性 $(\mathrm{S} 2)$ 、 服务水平 $(\mathrm{S} 3)$ [6]。

\section{3. 样本选择与计算方法}

本文以上海市市郊居民为研究对象, 对其进行了抽样 调查, 发放问卷 1000 份, 根据调查的需要, 剔除不符合要 求的问卷后, 实际有效问卷717份, 问卷调查有效回收率 $71.7 \%$, 满足置信区间 $95 \%$ 、相对误差 $5 \%$ 下样本量的要求。 其中, 被访男性占 $48 \%$, 女性占 $52 \%$, 样本覆盖各种职业、 出行目的和出行方式。

本文使用软件Smart PLS2.0分析对采集到的数据进行 实证分析。图1是利用SmartPLS建立的满意度评价模型:

(1) 黑色圆圈表示模型中的 7 个潜变量, 潜变量之间的数 值关系称为路径系数; (2) 长方形表示每个潜变量对应的 观测变量, 共通过 16 个观测变量来反映 7 个潜变量, 潜变 
量和观测变量之间的数值关系称为载荷系数, 数值越高, 说明观测变量的反映潜变量程度越高。

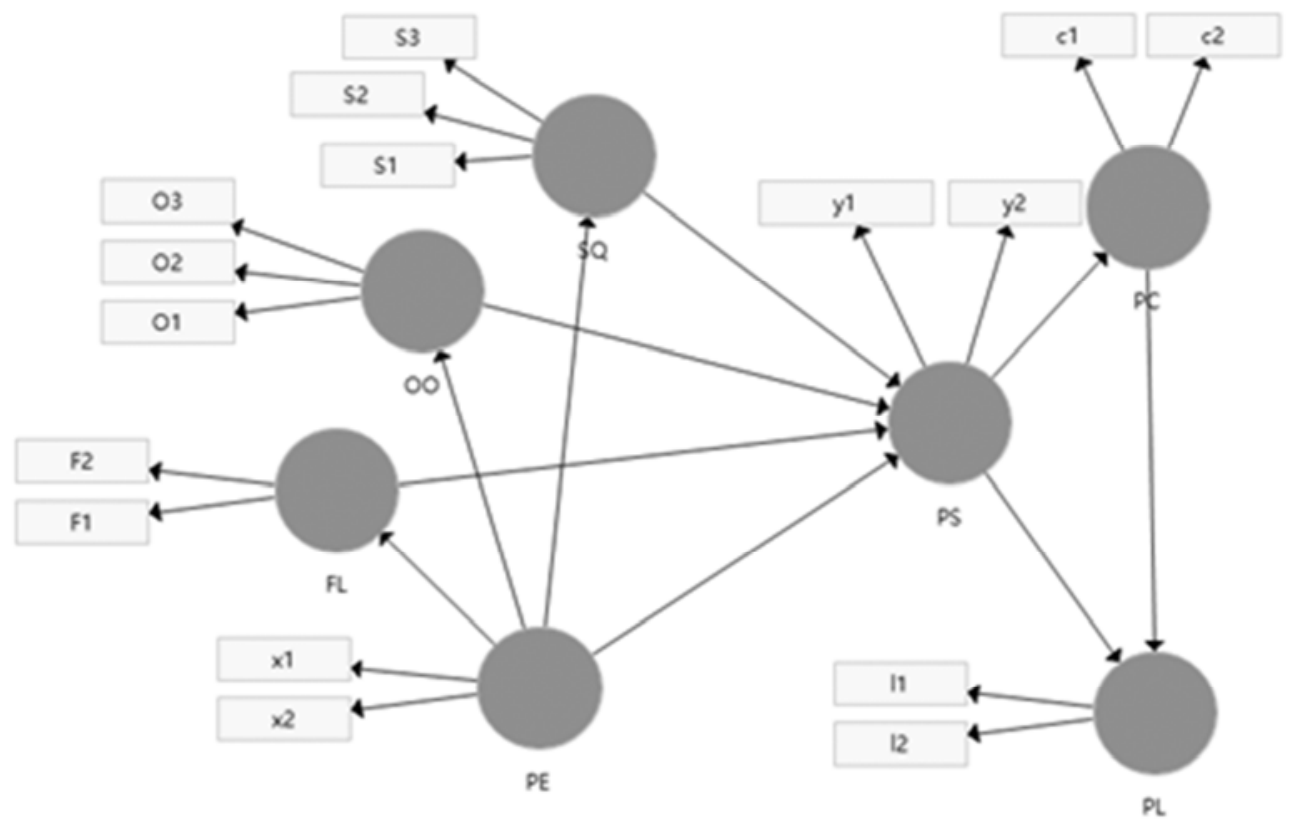

图1 出行满意度评价模型。

\section{4. 模拟计算与结果分析}

在数据分析前, 采用下面公式计算问卷的Cronbach's alpha系数, $\mathrm{k}$ 表示模型中可观测的指标总数, $\mathrm{S}_{\mathrm{i}}$ 表示第 $\mathrm{i}$ 个 指标的方差, $S_{X}$ 表示全部指标的总方差[5]:

$$
\alpha=\frac{k}{k-1}\left(1-\frac{\sum S_{i}^{2}}{S_{X}^{2}}\right)
$$

得到采用公交出行乘客的问卷Cronbach's alpha系数 为 0.787 , 说明问卷信度良好。利用问卷得到的实际数据, 计算各项所得平均分，平均分越高代表公众对该项指标的 评价越高。利用smartPLS软件对该模型计算求解, 得到各 潜变量之间的路径系数图 2 , 对应结构方程模型中 $\mathrm{R}^{2}=55.5 \%$, 说明模型可以解释 $55.5 \%$ 的结果, 解释能力超 过 0.33 , 模型具有整体的合理性 [5]。通过潜变量的组成信 度、Cronbach's alpha系数和平均萃取变异量评价模型的内 在结构适配度。

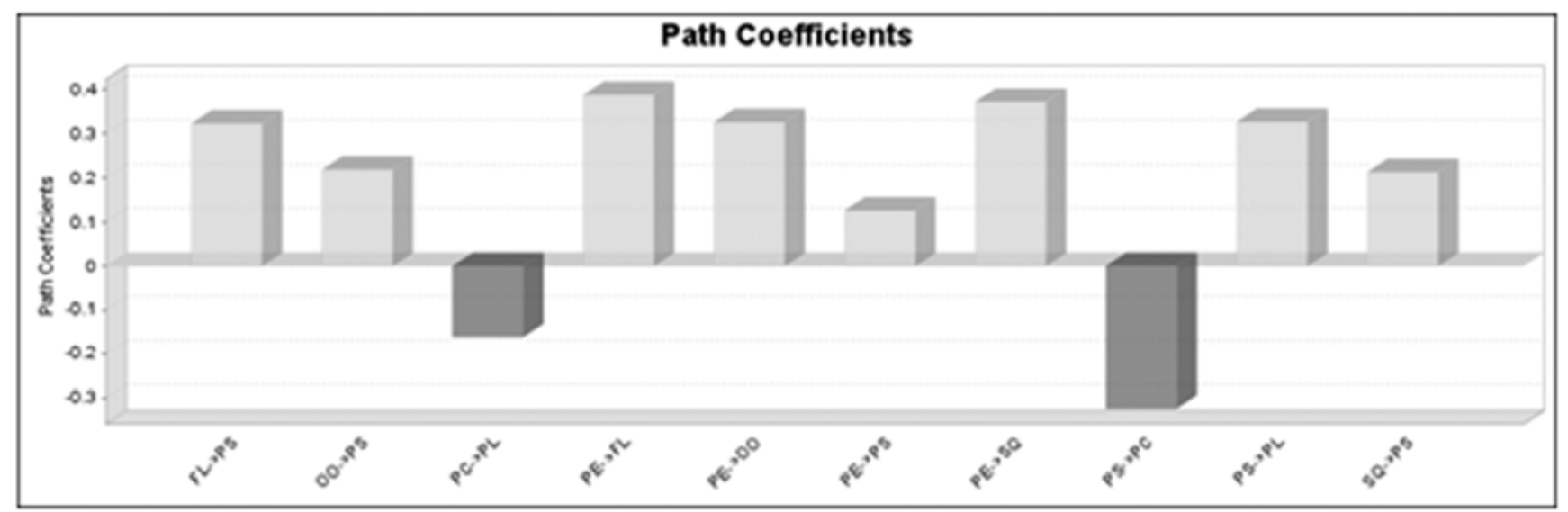

图2 出行满意度评价模型。

如表 1 所示, 除乘客抱怨 (PC) 组合信度略低于 0.7 外, 各潜变量组合信度都达到良好指标, 平均萃取变异量均超 过 0.5 , 说明整体模型结构适配度较好。模型中载荷系数 都大于 0.5 小于 0.95 , 且均在 0.05 显著性水平下显著, 说明 模型整体上符合适配标准。路径系数和载荷系数反应了一
个变量变化引起另一个变量变化的程度[1], 通过这些系统 的大小正负关系, 可以找出影响出行满意度的正面和负面 因素, 通过计算还可以的到优先改进系数, 找到优先改进 方向, 提高相关服务水平。 
表1 公交出行模型参数估计结果。

\begin{tabular}{|c|c|c|c|c|c|c|}
\hline 潜变量 & 观测变量 & 平均值 & 外部权重 & 载荷系数 & 组合信度 & 平均萃取变异量 \\
\hline \multirow{3}{*}{$\mathrm{FL}$} & F1 & 3.41 & 0.67 & 0.83 & \multirow{3}{*}{0.78} & \multirow{3}{*}{0.64} \\
\hline & F2 & 3.36 & 0.58 & 0.77 & & \\
\hline & O1 & 2.79 & 0.58 & 0.73 & & \\
\hline \multirow[t]{3}{*}{$\mathrm{OO}$} & $\mathrm{O} 2$ & 2.97 & 0.53 & 0.70 & \multirow[t]{3}{*}{0.70} & \multirow[t]{2}{*}{0.54} \\
\hline & O3 & 3.21 & 0.37 & 0.55 & & \\
\hline & S1 & 3.68 & 0.47 & 0.77 & & \multirow{3}{*}{0.58} \\
\hline \multirow[t]{2}{*}{ SQ } & S2 & 3.25 & 0.44 & 0.77 & \multirow[t]{2}{*}{0.80} & \\
\hline & S3 & 2.92 & 0.41 & 0.74 & & \\
\hline \multirow{2}{*}{ PC } & c1 & 0.70 & 0.80 & 0.73 & \multirow{2}{*}{0.66} & \multirow{2}{*}{0.50} \\
\hline & c2 & 0.29 & 0.60 & 0.70 & & \\
\hline \multirow{2}{*}{ PL } & 11 & 0.39 & 0.64 & 0.81 & \multirow{2}{*}{0.78} & \multirow{2}{*}{0.64} \\
\hline & 12 & 0.64 & 0.61 & 0.79 & & \\
\hline \multirow{2}{*}{ PE } & $\mathrm{x} 1$ & 3.69 & 0.54 & 0.77 & \multirow{2}{*}{0.79} & \multirow{2}{*}{0.67} \\
\hline & $\mathrm{x} 2$ & 3.88 & 0.68 & 0.86 & & \\
\hline \multirow{2}{*}{ PS } & yl & 3.34 & 0.61 & 0.80 & \multirow{2}{*}{0.79} & \multirow{2}{*}{0.65} \\
\hline & $\mathrm{y} 2$ & 3.28 & 0.63 & 0.81 & & \\
\hline
\end{tabular}

感知质量下载荷系数最高的三个指标为可达性 (F1)、 安全性 ( S1) 、便捷性 (F2), 这是公众最迫切需要解决 的问题, 说明调查区域线路设置不够合理、车辆行驶安全 性较差、便捷程度不够等实际情况吻合, 是下一步优化改 进的重点。设施水平 (FL) 中载荷系数最高的是等候时间 (O1), 说明在设施水平方面, 公众最关系的是等候车辆 的时间、发车频率等。以上通过外部模型, 由观测变量的 外部权计算得到各潜变量的值, 从而可以对公共交通系统 进行优化 [1]。

从模型的结果来看, 乘客期望 (PE)、设施水平 (FL)、 运营组织 $(\mathrm{OO})$ 、服务质量 $(\mathrm{SQ})$ 、乘客忠诚 (PL) 都 对乘客满意 (PS) 有正向解释能力, 其中设施水平 (FL) 对乘客满意 (PS) 的影响最大, 可能是因为在目前的公共 交通运营服务中设施建设还不够完善、充足, 很大程度上 反应了公众目前最需要关注的方面, 说明在调查区域内, 公交相关服务还存在大量缺口, 不能满足大家的需求。这
符合上海市市郊交通出行的现状, 站点覆盖率不够、便捷 程度不够。乘客满意 (PS) 对乘客抱怨 (PC) 有负向解 释能力, 乘客抱怨 (PC) 对乘客忠诚也具有负向解释能力, 说明它们之间呈负相关关系, 符合公众的一般认知。

同样的, 对于打车、使用共享单车或步行的公众出行, 参数估计结果见图3-5。

\section{1. 建立模型}

除感知质量潜变量的指标外, 其他 4 个潜变量与上述 公交系统潜变量指标完全相同。打车出行方感知质量设施 水平 (FL) 包含可达性 (F1)、便捷性 (F2), 运营组 织 (OO) 对应等候时间 ( $\mathrm{O} 1)$ 、服务规范 (O2) 和服务 范围（O3）、服务质量（SQ）对应指标有便捷性（S1）、 安全性 (S2)、舒适性 (S3)。

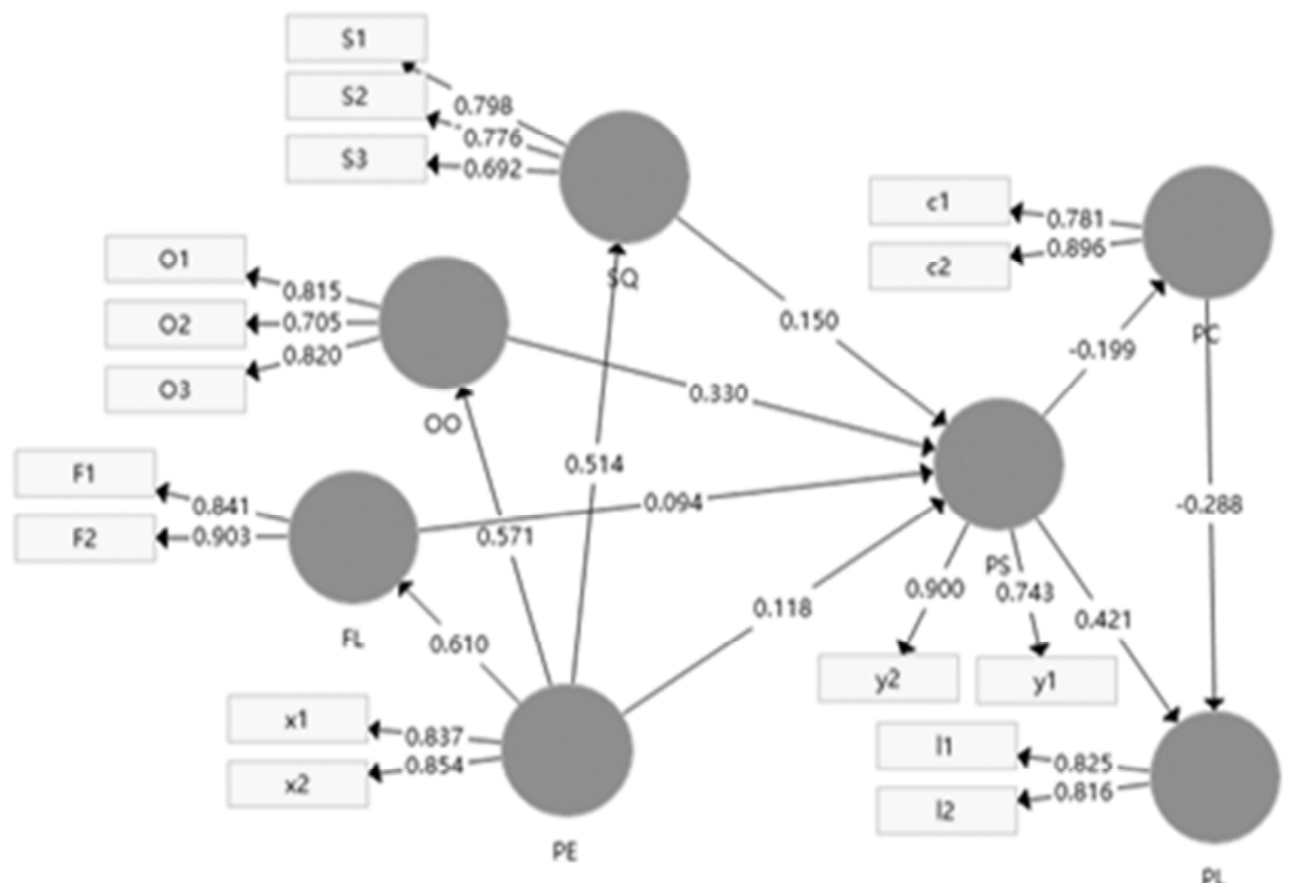

图3 打车出行满意度参数估计结果。 
共享单车出行方感知质量设施水平（FL）包含可获得性（F1）、便捷性（F2），运营组织（OO）对应维护效率 $(\mathrm{O} 1)$ 、收费水平 $(\mathrm{O} 2)$ 和软件运营（O3）、服务质量（SQ）对应指标有便捷性（S1）、安全性（S2）。

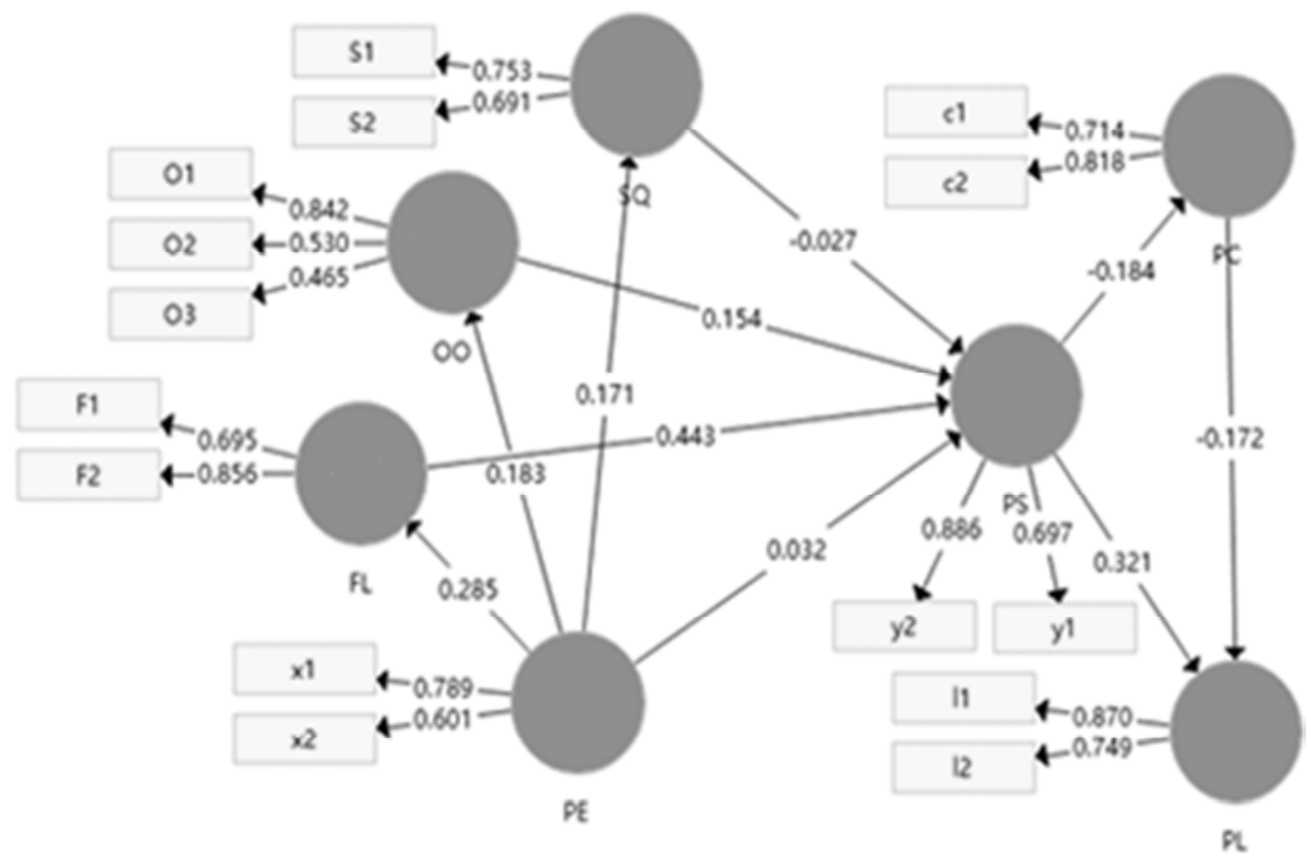

图4 共享单车出行满意度参数估计结果。

步行出行方感知质量设施水平（FL）包含可达性（F1）、线路便捷（F2）、设施齐全（F3），运营组织（OO） 对应等候时间（O1）、维护效率（O2）、服务质量（SQ）对应指标有舒适性（S1）、卫生性（S2）、安全性（S3）、 服务水平（S4）。

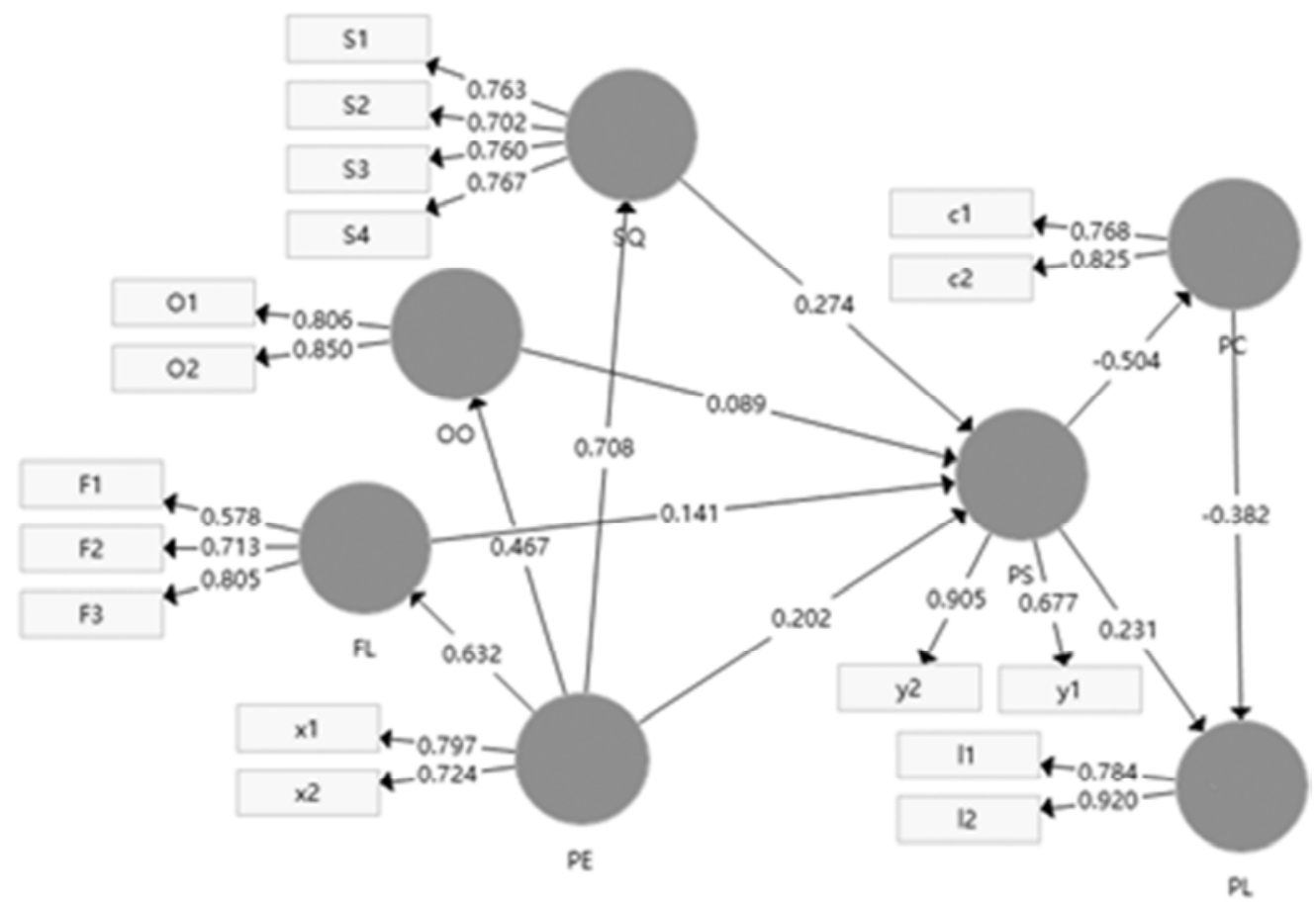

图5 步行出行满意度参数书估计结果。

\section{2. 信度检验与模型拟合}

分别对四种典型出行方式进行信度检验, 如表 2 所示。检验结果Cronbach' s alpha系数均大于 0.7 , 信度较好。 
表2 四种出行方式的Cronbach's alpha系数。

\begin{tabular}{ll}
\hline 出行方式 & Cronbach's alpha系数 \\
\hline 公交 & 0.787 \\
打车 & 0.841 \\
共享单车 & 0.706 \\
步行 & 0.838 \\
\hline
\end{tabular}

四种出行方式的模型拟合指数如表 3 所示, 均大于 0.33 , 解释能力尚可。

表3 四种出行方式的模型拟合指数。

\begin{tabular}{ll}
\hline 出行方式 & $\mathbf{R}^{2}$ \\
\hline 公交 & $55.5 \%$ \\
打车 & $44.6 \%$ \\
共享单车 & $38.9 \%$ \\
步行 & $46.6 \%$ \\
\hline
\end{tabular}

\section{3. 载荷系数}

利用下面满意度指数公式计算各种出行方式的满意 度指数 $[4]$ :

$$
\text { 满意度系数 }=\frac{E(\eta)-\min (\eta)}{\max (\eta)-\min (\eta)}
$$

式中, $\eta$ 表示某一个潜变量。90 100分表示非常满意, $80 \sim 90$ 分表示比较满意, $70 \sim 80$ 分表示一般满意, $60 \sim 70$ 分表示较不满意, 60 分以下表示非常不满意[4]。再利用各 交通出行方式的权重, 可以计算得到“最后一公里”交通运 输系统的总体满意度。从表 4 可以看出, 公众对市郊出行 的总体满意度较低, 尤其是对共享单车的满意度最低, 与 目前共享单车的投放策略相吻合, 反映了目前交通运输系 统极不完善, “最后一公里”交通运输系统的建设非常迫切, 非常关键。

表4 四种出行方式的满意度指数。

\begin{tabular}{llllll}
\hline 出行方式 & 观测变量 & 平均值 & 载荷系数 & 满意度指数 & 总满意度指数 \\
\hline 公交 & $\mathrm{y} 1$ & 3.34 & 0.80 & 57.74 & \\
& $\mathrm{y} 2$ & 3.28 & 0.81 & 61.23 & 58.18 \\
打车 & $\mathrm{y} 1$ & 3.34 & 0.74 & 55.52 & \\
& $\mathrm{y} 2$ & 3.54 & 0.90 & & \\
共享单车 & $\mathrm{y} 1$ & 3.19 & 0.89 & 5.70 & \\
步行 & $\mathrm{y} 2$ & 3.26 & 0.90 & 0.68 & \\
\hline
\end{tabular}

\section{5. 结论}

本文建立的城轨接驳换乘满意度评价模型中有乘客 期望（PE）、乘客满意（PS）、乘客抱怨（PC）、乘客 忠诚 $(\mathrm{PL})$ 、设施水平 $(\mathrm{FL})$ 、运营组织 $(\mathrm{OO})$ 和服务 质量 $(\mathrm{SQ})$ 共7个变量, 结构变量与乘客满意度指数模型 相适应, 并且根据出行方式的不同, 调整相应观测变量。 具体应用时, 可根据不同交通方式的特点和考查目的调整 模型, 并且针对测评指标进行优化。

本文提出的满意度评价模型为综合交通运输系统的 评价提供了新的工具, 也可为其他领域的满意度评价提供 新思路。从实证情况看, 该模型通用性、可移植性非常强, 能够应用于多种交通系统, 分析复杂网络中各变量的相互 关系, 可充分有效地利用原始数据中的有效信息，获得影 响满意度评价的关键因素。并且模型变量设置方便直接, 更符合公众认知, 也可以减少采集数据的难度。

\section{致谢}

本文为同济大学大学生创新实践训练计划《地铁乘客 的最后一公里满意度调查及质量提升对策》的阶段性成果 之一。

\section{参考文献}

[1] GB/T 19038-2009 顾客满意测评模型和方法指南[S]。

[2] Cardozo R N. An Experimental Study of Customer Effort, Expectation, and Satisfaction[J]. Journal of Marketing Research, 1965, 2(3):244-249.

[3] 方蕾,邹哲,庞志显. 城市轨道交通服务质量模糊综合评判 [J]. 城市, 2004(1):28-30。

[4] 张双. 地铁乘客满意度评价体系研究 [D]. 西安: 长安大学, 2008.

[5] 吴绍玉. 基于 CCSI的 T市地铁乘客满意度研究 [D]. 天津: 天津大学, 2012.

[6] 金宁.公共交通乘客满意度测评理论及实证研究[D].吉林大 学,2009.

[7] 顾品. 基于顾客满意度的无锡地铁服务质量评价研究 [D]. 华东政法大学, 2015 .

[8] 郭陆灯,李博,王佳,等. 基于轨道换乘满意度的结构方程模型 建立 [J].长春理工大学学报(自然科学版),2017, 40(2):37-40.

[9] Smith R G W, Mulamoottil G. The access journey to the railway station and its role in passengers' satisfaction with rail travel[J]. Transport Policy, 2007, 14(5):357-365. 
[10] 乔婧,孙立山,刘小明,等.基于结构方程的公共交通换乘影响 因素研究[J].科学技术与工程, 2017, 17(6):296-300.

[11] 周强.地铁接驳的公共自行车使用特征与主观幸福感研究 [D].苏州大学, 2015 .

[12] 滕靖,段帅,李建琳,等.浅议城市轨道交通乘客满意度评价方 法应用[J]. 华东公路, 2017(3):100-103.
[13] 袁玲.城市地铁服务质量评价研究 [D]. 南京航空航天大 学, 2013 .

[14] 尤雨婷. 轨道交通与短距离支撑方式接驳特性及资源配置 优化[D].东南大学, 2016.

[15] 莫一魁,沈旅欧.城市公交系统公众评价的偏最小二乘通径 模型[J]. 深圳大学学报(理工版), 2009, 26(4):436-440. 